\title{
Epidemiology of Alcohol Misuse and Illicit Drug Use Among Young People Aged 15-24 Years in Fishing Communities in Uganda
}

\author{
Monica O. Kuteesa ${ }^{1,2, *}$, Helen A. Weiss ${ }^{3}$, Sarah Cook ${ }^{4}$, Janet Seeley ${ }^{1,5}$, Josephine N. Ssentongo ${ }^{1}$, \\ Robert Kizindo ${ }^{1}$, Paul Ngonzi ${ }^{1}$, Moses Sewankambo ${ }^{1}$ and Emily L. Webb ${ }^{3}$ \\ 1 MRC/UVRI and LSHTM Uganda Research Unit, Entebbe P.O.Box 49 Entebbe, Uganda; \\ janet.seeley@lshtm.ac.uk (J.S.); Josephine.Naluwugge@mrcuganda.org (J.N.S.); \\ robert.kizindo@mrcuganda.org (R.K.); pngonzi@gmail.com (P.N.); \\ moses.sewankambo@mrcuganda.org (M.S.) \\ 2 Department of Infectious Disease Epidemiology, London School of Hygiene and Tropical Medicine, \\ Keppel Street, London WC1E 7HT, UK \\ 3 Medical Research Council Tropical Epidemiology Group, London School of Hygiene and Tropical \\ Medicine, London WC 1E, UK; helen.weiss@lshtm.ac.uk (H.A.W.); emily.webb@lshtm.ac.uk (E.L.W.) \\ 4 Department of Non-Communicable Disease Epidemiology, London School of Hygiene and Tropical \\ Medicine, London WC 1E, UK; sarah.cook@lshtm.ac.uk \\ 5 Department of Global Health and Development, London School of Hygiene and Tropical Medicine, \\ London WC1E 7HT, UK \\ * Correspondence: monica.kuteesa@lshtm.ac.uk; Tel.: +256-417-704-000
}

Received: 11 March 2020; Accepted: 30 March 2020; Published: 1 April 2020

\begin{abstract}
Background: We determined the prevalence of and risk factors for alcohol misuse and illicit drug use among young Ugandans in fishing communities, a recognised "key population" for human immunodeficiency virus (HIV) infection. Methods: We conducted a cross-sectional survey among young people (15-24 years) in fishing communities in Koome, Uganda, in December 2017July 2018. Using Audio-Assisted Self-Interviewing, we collected data on socio-demographic characteristics and alcohol use, including the Alcohol Use Disorders Identification Test (AUDIT) and timeline follow-back calendar (TLFB). Blood samples were analysed for HIV, herpes simplex virus 2 (HSV2), and Phosphatidyl ethanol (PEth 16:0/18:1). Urine samples were analysed for illicit drugs. Results: Among 1281 participants (52.7\% male, mean age 20 years), 659 (51.4\%) reported ever drinking alcohol, 248 (19.4\%) had 12-month-AUDIT $\geq 8$, and 261 (20.5\%) had whole-blood PEth 16:0/18:1 concentration $\geq 20 \mathrm{ng} / \mathrm{mL}$, indicating significant consumption. In multivariable analyses, PEth 16:0/18:1 $\geq 20 \mathrm{ng} / \mathrm{mL}$, AUDIT $\geq 8$ and binge drinking ( $\geq 6$ standard drinks per drinking occasion in the previous month from TLFB) were all strongly associated with older age, low education, smoking, and HSV2. Illicit drug use prevalence was $5.2 \%$ and was associated with older age, low education, being single, and smoking. Conclusion: Levels of alcohol misuse were high among young people in fishing communities and associated with HSV2, a proxy for risky sexual behaviour. Alcohol and illicit drug harm reduction services and HIV prevention programs in Uganda should prioritise young fisherfolk.
\end{abstract}

Keywords: alcohol; drugs; substance use; fisherfolk; adolescents; survey; Sub-Saharan Africa

\section{Introduction}

Alcohol and illicit drug use are major global risk factors for disability and premature mortality and contribute to both communicable and non-communicable diseases [1,2]. This health burden is 
accompanied by significant economic costs, including expenditure on health care and law enforcement, loss of productivity and other direct and indirect costs including harm to others $[1,2]$. Adolescence is a vulnerability window for initiation and continuation of polysubstance use and associated harms [3]. For instance, globally, the prevalence of current drinking increases from $26.5 \%$ among 15-19 year olds to $40.7 \%$ among 20-24 year olds, while the prevalence of heavy episodic drinking increases from $13.6 \%$ to $21.8 \%$ [1]. An estimated $14 \%$ of all deaths among $20-39$ year olds are attributable to alcohol [4].

The Sub-Saharan Africa (SSA) region has the highest estimated prevalence of heavy episodic drinking per drinker globally (25\%), high levels of alcohol related disease and injury, large amounts of unrecorded consumption of illegally produced alcohol [1], and a growing market for alcohol sales [5]. An estimated 5.6\% of young people aged 15-19 years (13.8 million) used cannabis in 2015 [4] and there are increasing reports of opioid and injection drug use [6]. Concurrent with the apparent increase in substance use in SSA, human immunodeficiency virus (HIV) remains a major public health problem, with young women aged 15-24 years and people who inject drugs disproportionately affected [7]. In a recent systematic review, we showed that in SSA, information on the burden of alcohol misuse and illicit drug use is limited, especially among young people belonging to certain occupational groups (i.e., sex workers, miners, truckers, fishing communities, uniformed personnel and motorcycle taxi riders) known to be at increased risk of HIV acquisition [8-13].

Fishing communities are a recognised key population group in Uganda, and some evidence suggests that as well as being at increased risk of HIV acquisition [10,14], they may also be at increased risk for substance use due to a combination of factors, including occupational norms, high mobility $[15,16]$, geographical remoteness and inadequate substance use regulation $[17,18]$. However, studies on the burden of alcohol misuse remain few and diagnosis is challenging, as most studies have not used locally validated tools. The aim of this paper is to estimate the prevalence and correlates of alcohol misuse and illicit drug use, respectively, and their association with HIV risk behaviour, among young people in fishing communities on Lake Victoria in Uganda.

\section{Materials and Methods}

\subsection{Study Setting, Design and Procedures}

Between December 2017 and July 2018, we conducted a cross-sectional survey in fishing communities on the Koome Islands in Mukono District, Uganda, with an estimated population of 16,000. HIV prevalence in Koome was around 21\% in adults aged 18+ in 2016 [19]. Based on data from a household survey conducted in 2013, as part of the LaVIISWA study [20], within the 15-25 year age group, reported alcohol misuse (defined as consuming at least five alcoholic drinks per day in the last 12 months on average), prevalence was $5 \%-6 \%$.

The target population was young people aged 15-24 years and the primary outcome was prevalence of alcohol misuse. The target sample size of 1204 young people was calculated to allow us a precision of $2.5 \%$ with which to estimate a prevalence of alcohol misuse of up to $15 \%$ [19,21], assuming a design effect of 1.5 . If the design effect was higher, at 2 , then this sample size would allow us precision of $2.5 \%$ with which to estimate a prevalence of up to $11 \%$. The target sample size would also provide $80 \%$ power to detect a difference in HIV/herpes simplex virus 2 (HSV2) prevalence of $20 \%$ versus $8 \%$ in young people who misuse alcohol compared to those who do not, assuming that prevalence of alcohol misuse is $12.5 \%$, and the design effect is 2 .

We used a two-stage sampling strategy, yielding a self-weighted sample. First, 20 villages were selected from the 27 villages on the islands using simple random sampling. Pre-existing village lists of households (defined as people who sleep in the same house and share meals) were available, but there was no list of individuals to use as a sampling frame. Therefore, we used the households as our sampling unit. We updated the village's lists of households, and then sampled households from each selected village. Each household had a probability of 0.69 of selection; this probability was calculated to achieve the required sample size based on prior knowledge of the approximate number of young people per household. This approach ensured that all households across the study setting had equal 
probability of selection. We further assumed that the number of young people per household was consistent across the study setting. We included all young people in the selected households in our survey. This approach yielded a self-weighted sample. Young people aged 15-24 years and resident in the selected households were invited to participate and were enrolled after providing written informed consent. Non-emancipated minors gave assent and their parents/guardians provided parental consent. We excluded severely ill persons.

\subsection{Data Collection}

Survey data were collected by five trained interviewers (three psychiatry clinicians and two experienced non-clinical interviewers), privately, at the community hub or another agreed location. A questionnaire including items on socio-demographic characteristics of study participants, alcohol, tobacco and illicit drug use, depression, and sexual behaviour was developed. It was translated into the local language and back-translated into English separately by two trained translators; versions were compared, and necessary adjustments made to ensure accuracy. Most young people in Koome speak Luganda or English and the ability to do so was an inclusion criterion. All questions were then reviewed by community groups of adolescents from our target population for conceptual understanding and translation accuracy.

The questionnaire was administered using Audio Computer Assisted Self-Interviewing (ACASI). Respondents completed the questionnaire using a tablet and headphones, reading on their own and hearing a recorded voice speak the questions and response options. To build ACASI, both the English and Luganda versions of the questionnaire were audio recorded and programmed into the tablet by the research team, with skip patterns for questions that were not relevant. The questionnaire was pilot tested on 30 young people in a separate village in the same study area. Data collected from the pilot village were not included in this analysis; the results of the pilot were only used to modify survey collection instruments, field procedures, and data management systems, as necessary. During data collection, participants were provided with brief training on how to use the tablets and research staff were stationed nearby to answer questions and provide assistance, as necessary. Study participants were able to complete the interview in the language of their choice.

\subsection{Measures}

Demographic measures included age, religion, education, tribe, village, occupation and parity. Harmful alcohol use was assessed using the following measures. (i) The Alcohol Use Disorders Identification Test (AUDIT) - a 10-item screening tool developed by the World Health Organisation (WHO) to screen for harmful or hazardous drinking. An AUDIT score of $\geq 8$ is considered to indicate hazardous or harmful alcohol use. The AUDIT has been validated across genders and in a wide range of racial/ethnic groups and is well-suited for use in primary care settings [22]. (ii) The timeline followback (TLFB) tool-a calendar method that can be used as a clinical and research tool to obtain a variety of quantitative estimates of alcohol or illicit drug use patterns over a specified time period that can vary from daily use up to 12 months from the interview date [23]. (iii) Phosphatidyl ethanol (PEth), a more sensitive and specific biomarker compared to traditional biomarkers (gammaglutamyl transpeptidase (GGT), mean corpuscular volume (MCV) and carbohydrate-deficient transferrin (CDT)), for detecting chronic heavy drinking. PEth is detectable in whole blood for more than two weeks [24], and PEth $\geq 10 \mathrm{ng} / \mathrm{mL}$ has been shown to be highly sensitive (88\%) and specific (89\%) for any alcohol consumption among Ugandan adults living with HIV, in the prior 21 days [25]. Both AUDIT and TLFB tools were administered using ACASI while PEth was determined in dry blood spot (DBS) samples. Alcohol misuse based on AUDIT was defined as a score $\geq 8$. Binge drinking based on TLFB was defined as $\geq 6$ standard drinks per drinking occasion in the previous month. For this study, illicit drug use is defined as use of a substance where consumption has been prohibited by international drug control treaties except for medical purposes [26], and was assessed using urine tests. We also collected self-reported data on illicit drug use using the Alcohol, Smoking and Substance Involvement Screening Test (ASSIST), a screening tool recommended by the WHO to detect and manage substance use and related problems in primary and general medical care settings; 
it has been found to be feasible and have adequate test-retest reliability among adult populations in several geographic locations [27-29]. Depression severity was measured using the Patient Health Questionnaire (PHQ-9) [30]. Sexual risk behaviour was assessed by HSV2 and HIV results.

\subsection{Laboratory Testing}

To measure PEth, we used ethanol-free swabs for disinfection of the venous puncture site and collected capillary DBS samples. DBSs were dried at room temperature, without any direct sun, for three hours and stored in an airtight mini-grip bag with a drying agent in a fridge at $<8^{\circ} \mathrm{C}$ for up to 4 days. DBSs were then transported to the MRC/UVRI and LSHTM Uganda Research Unit reference laboratory in Entebbe and stored at $-20^{\circ} \mathrm{C}$ prior to express delivery on dry ice to the University of Bern, Switzerland, for analysis. DBSs were kept at $-20^{\circ} \mathrm{C}$ in the Bern laboratory and analysed within five to seven days of arrival to prevent in-vitro formation of PEth. Assays of PEth 16:0/18:1 and 16:0/18:2, the main PEth homologues in human blood [31] were conducted using high-performance liquid chromatography-tandem mass spectrometry (LC-MS/MS), using selected ion monitoring (SIM) in the negative mode of the deprotonated molecules. Here we report on PEth 16:0/18:1. Cutoffs for evaluating PEth 16:0/18:1 values were light or no consumption $(<20 \mathrm{ng} / \mathrm{mL})$, significant consumption (20-209 $\mathrm{ng} / \mathrm{mL})$ and heavy consumption $(\geq 210 \mathrm{ng} / \mathrm{mL})[32]$.

Additional laboratory tests included using a pre-defined nationally approved standard serial testing algorithm for HIV [33]: Alere Determine HIV 1/2 whole blood assay (Alere medical, Chiba, Japan) for screening followed by STAT-PAK rapid test HIV 1/2 (Chembio diagnostic systems New York, U.S.A) as confirmatory test and SD Bioline (Standard Diagnostics, Inc., Kyonggi-do, South Korea) as the tie breaker. For discordant results, confirmatory tests were conducted in an accredited laboratory using ELISA HIV (Murex, Diasorin: Ref 9E25-02, Saluggia VC, Italy) and Bio Elisa HIV1/2 $\mathrm{Ag} / \mathrm{Ab}$ (BIOKIT, Barcelona, Spain). Newly diagnosed participants were referred for antiretroviral therapy initiation. HSV2 testing was performed at the Entebbe research laboratory using the Kalon ELISA (Kalon Biological, UK) with a cut-off optical density of 1.5. Fresh urine samples collected from all participants were tested at the study site for illicit drugs using a the (one-step rapid test, Protzek (Lörrach, Baden-Württemberg, Germany). This multiplate test is a sensitive immunochromatographic test based on a specific antigen-antibody reaction for the qualitative detection of amphetamines, methamphetamines, cocaine, ecstasy, opiates, and cannabinoids in human urine.

\subsection{Data Management and Statistical Analysis}

Data were captured electronically using an open data kit (ODK) and checked for missing fields and erroneous inputs. All statistical analyses were conducted using Stata, version 15 (Stata Corp, College Station, Texas, USA). We summarised participants' socio-demographic characteristics and conducted univariable and multivariable analyses to identify factors associated with alcohol misuse (four separate outcomes: AUDIT $\geq 8$, PEth $\geq 20 \mathrm{ng} / \mathrm{mL}$, PEth $\geq 210 \mathrm{ng} / \mathrm{mL}$, binge drinking based on TLFB) and illicit drug use. Multivariable analysis to control for potential confounding was guided by a hierarchical conceptual framework (Figure 1), following the approach described by Victora et al. [34]. We classified each variable into one of three groups (levels) from most distal to most proximal to the outcomes of interest for this analysis (alcohol misuse and illicit drug use). Level 1 variables were those determined at birth or during childhood, level 2 variables comprised current sociodemographic variables, and level 3 variables comprised current behaviour factors and infections. Level 1 variables were thought likely to impact on the outcomes through their effects on level 2 and hence on level 3 variables. Therefore, when assessing the effect of level 1 variables on the outcomes, we did not adjust for level 2 or level 3 variables in order to avoid adjusting for factors on the causal pathway. Similarly, when assessing the effect of level 2 variables on the outcomes, we did not adjust for level 3 variables. In summary, variables at the first level were adjusted for each other, variables at the second level were adjusted for each other and for first level variables, and third level variables were adjusted for each other and for first and second level variables. The only exception to this was that results for HIV were not adjusted for HSV2 and vice versa, since the two were both considered 
proxies for sexual behaviour and are highly correlated. Variables were retained in models regardless of their p-value after adjustment following this strategy; they were not excluded on the basis of high p-values. This strategy allowed us to assess the effects of variables at each level of the conceptual framework, having adjusted for more distal variables [34]. We used a two-stage sampling strategy and the sample was self-weighted. We accounted for clustering at the village and household level: logistic regression with a robust variance implemented using the svyset command in Stata was used to assess risk factors for alcohol misuse and illicit drug use. 


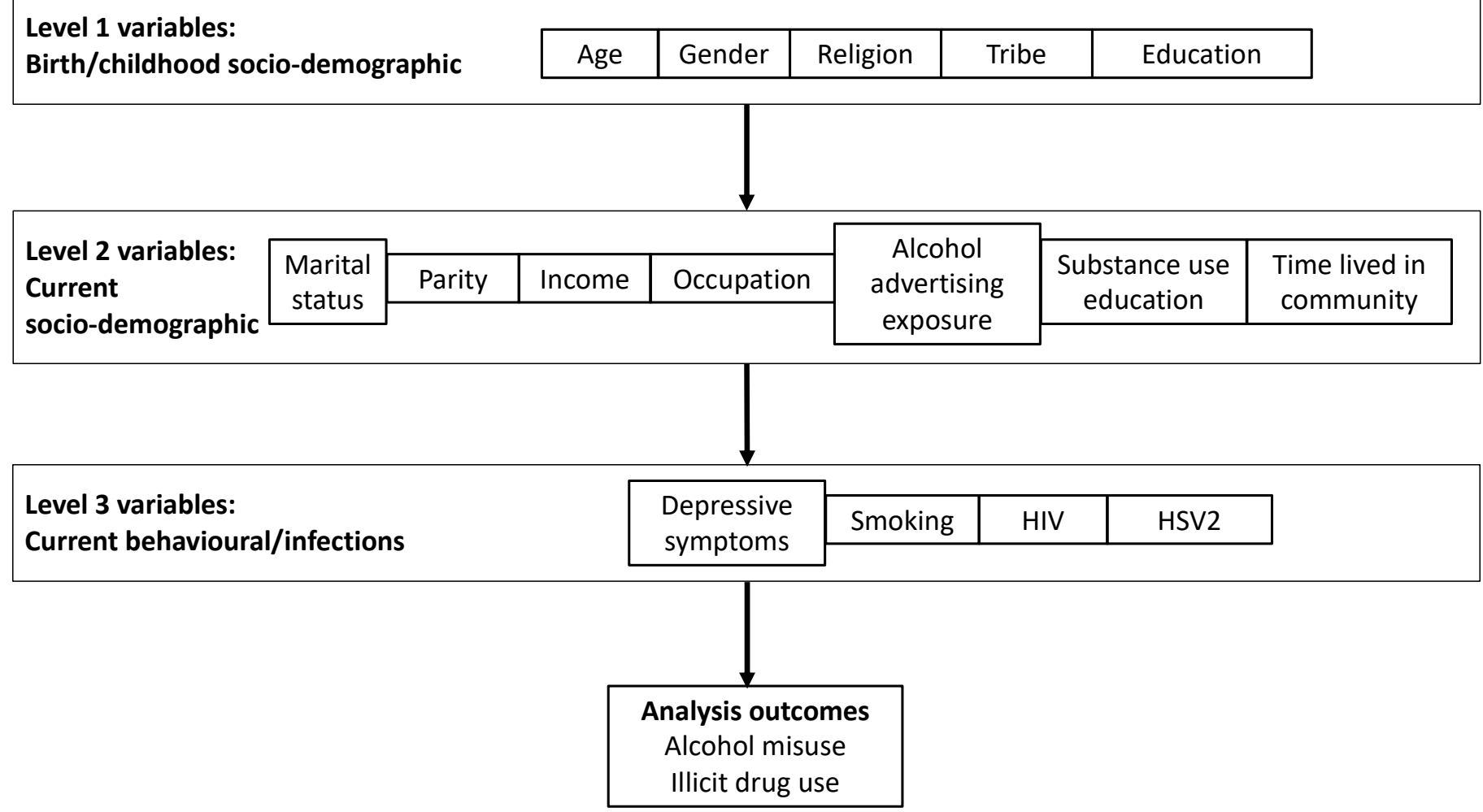

Figure 1. Conceptual framework for determinants of alcohol misuse and illicit drug use. HIV-Human Immunodeficiency Virus, HSV2-herpes Simplex Virus. 


\subsection{Ethics Statement}

We obtained ethical approval from the Uganda Virus Research Institute research and ethics committee (Ref: GC/127/17/07/595, approved on 27th July 2012), the London School of Hygiene and Tropical Medicine ethics committee (Ref: 14299, approved on 3rd October 2017), and the Uganda National Council for Science and Technology (Ref: SS 4385, approved on 6th October 2017) and the office of the president (Ref: ADM 194/212/01, approved on 20th October 2017). Any participants requiring medical treatment were referred to nearby government health centres where free treatment was offered.

\section{Results}

A total of 4521 households in the 20 villages were randomly selected for inclusion in the survey. Of these, 1115 were occupied by at least one young person aged 15-24 years. Residents of 146 households (representing 340 individuals) did not take part in the study, with not being located during the survey period $(n=144)$ and refusals $(n=196)$ being the most common reason for nonparticipation. A total of 1281 eligible young people from 969 households participated in the survey. Administration of ACASI questionnaires was acceptable and feasible; only two participants had difficulty completing the ACASI and were interviewed face-to-face. The final sample comprised 1281 young people aged $15-24$ years (52.7\% male; mean age 20 years (SD 2.7). The majority $(61.3 \%)$ had attained primary education or less; $557(43.5 \%)$ were single, with more females $(253,41.8 \%)$ reporting being married than males $(158,23.4 \%)$. Approximately $43 \%$ of the sample had lived in the fishing community for less than six months. Sixty-seven participants (5.2\%) were living with HIV (1.8\% male, $9.1 \%$ female) and $406(32.0 \%)$ tested HSV2 positive (20.4\% male, $44.9 \%$ female) (Table 1: participant characteristics). Missing values include participants who did not provide blood samples $(n=7)$; for the very small number of other missing results (HIV $n=2, \operatorname{HSV} 2 n=4$, PEth $n=5$ ), the lack of results was a laboratory-based issue. 
Table 1. Participant characteristics.

\begin{tabular}{|c|c|c|c|c|c|c|c|}
\hline \multirow[t]{2}{*}{ Characteristics } & \multirow[t]{2}{*}{ Categories } & \multicolumn{2}{|c|}{ Men $(n=675)$} & \multicolumn{2}{|c|}{ Women $(n=606)$} & \multicolumn{2}{|c|}{ Overall $(n=1281)$} \\
\hline & & $\mathrm{n}$ & $\%$ & $\mathbf{N}$ & $\%$ & $\mathbf{N}$ & $\%$ \\
\hline \multirow[t]{2}{*}{ Age } & $15-19$ years & 261 & 38.7 & 226 & 37.3 & 487 & 38.0 \\
\hline & 20-24 years & 414 & 61.3 & 380 & 62.7 & 794 & 62.0 \\
\hline \multirow[t]{3}{*}{ Religion } & Christian & 505 & 74.8 & 461 & 76.1 & 966 & 75.4 \\
\hline & Muslim & 156 & 23.1 & 145 & 23.9 & 301 & 23.5 \\
\hline & Traditional/other & 14 & 2.1 & 0 & 0 & 14 & 1.1 \\
\hline \multirow[t]{4}{*}{ Marital status } & Married & 158 & 23.4 & 253 & 41.8 & 411 & 32.1 \\
\hline & Cohabiting & 106 & 15.7 & 118 & 19.5 & 224 & 17.5 \\
\hline & Single & 378 & 56.0 & 180 & 29.7 & 558 & 43.6 \\
\hline & Separated/divorced/widowed & 33 & 4.9 & 55 & 9.1 & 88 & 6.9 \\
\hline \multirow[t]{5}{*}{ Education attainment } & None & 22 & 3.3 & 15 & 2.5 & 37 & 2.9 \\
\hline & Incomplete primary & 338 & 50.1 & 262 & 43.2 & 600 & 46.8 \\
\hline & Complete primary & 76 & 11.3 & 73 & 12.1 & 149 & 11.6 \\
\hline & Secondary junior & 210 & 31.1 & 230 & 38 & 440 & 34.4 \\
\hline & Secondary senior and above & 29 & 4.3 & 26 & 4.3 & 55 & 4.3 \\
\hline \multirow{5}{*}{ Time resident in fishing community } & $<6$ months & 254 & 37.6 & 296 & 48.8 & 550 & 42.9 \\
\hline & 7 to 11 months & 114 & 16.9 & 100 & 16.5 & 214 & 16.7 \\
\hline & 1 to 5 years & 221 & 32.7 & 170 & 28.1 & 391 & 30.5 \\
\hline & 6 to 10 years & 37 & 5.5 & 16 & 2.6 & 53 & 4.1 \\
\hline & $>11$ years & 49 & 7.3 & 24 & 4 & 73 & 5.7 \\
\hline \multirow[t]{2}{*}{ Human Immunodeficiency Virus (HIV *) } & Positive & 12 & 1.8 & 55 & 9.1 & 67 & 5.3 \\
\hline & Negative & 657 & 98.2 & 548 & 90.9 & 1205 & 94.7 \\
\hline \multirow[t]{2}{*}{ Herpes Simplex Virus (HSV2 **) } & Positive & 136 & 20.4 & 270 & 44.9 & 406 & 32.0 \\
\hline & Negative & 532 & 79.6 & 332 & 55.2 & 864 & 68.0 \\
\hline \multirow[t]{2}{*}{ Ever used alcohol } & Yes & 363 & 53.8 & 296 & 48.8 & 659 & 51.4 \\
\hline & No & 312 & 46.2 & 310 & 51.2 & 622 & 48.6 \\
\hline \multirow[t]{5}{*}{$\begin{array}{l}\text { 30-day Alcohol Use Disorders Identification Test } \\
\text { (AUDIT) }\end{array}$} & Mean (SD) & 5.5 & 8.0 & 3.7 & 6.3 & 4.7 & 7.3 \\
\hline & $<7$ Low risk or non-drinker & 480 & 71.1 & 492 & 81.2 & 972 & 75.9 \\
\hline & 8-15 Excess of low risk & 105 & 15.6 & 70 & 11.6 & 175 & 13.7 \\
\hline & 16-19 Harmful drinking & 28 & 4.2 & 18 & 3.0 & 46 & 3.6 \\
\hline & $\geq 20$ Alcohol dependence & 62 & 9.2 & 26 & 4.3 & 88 & 6.9 \\
\hline
\end{tabular}




\begin{tabular}{|c|c|c|c|c|c|c|c|}
\hline \multirow[t]{5}{*}{ 12-month AUDIT } & Mean (SD) & 4.7 & 7.7 & 3.3 & 6.2 & 4.0 & 7.0 \\
\hline & $<7$ low risk or non-drinker & 530 & 78.5 & 503 & 83 & 1033 & 80.6 \\
\hline & 8-15 Excess of low risk & 72 & 10.7 & 60 & 9.9 & 132 & 10.3 \\
\hline & 16-19 Harmful drinking & 19 & 2.8 & 24 & 4 & 43 & 3.4 \\
\hline & $\geq 20$ Alcohol dependence & 54 & 8 & 19 & 3.1 & 73 & 5.7 \\
\hline \multirow{3}{*}{$\begin{array}{l}\text { Phosphatidylethanol (Peth) cut-off for alcohol use } \\
\text { (PEth 16:0/18:1) }\end{array}$} & Light or no consumption & 505 & 75.4 & 510 & 84.2 & 1015 & 79.5 \\
\hline & Heavy drinking $(20-209 \mathrm{ng} / \mathrm{mL})$ & 128 & 19.1 & 78 & 12.9 & 206 & 16.1 \\
\hline & Hazardous drinking $(\geq 210 \mathrm{ng} / \mathrm{mL})$ & 37 & 5.5 & 18 & 3.0 & 55 & 4.3 \\
\hline \multirow{3}{*}{$\begin{array}{l}\text { Timeline Followback(TLFB) ( } \geq 6 \text { standard drinks per } \\
\text { drinking occasion, in previous month) }\end{array}$} & & & & & & & \\
\hline & No & 615 & 91.1 & 585 & 96.5 & 1200 & 93.7 \\
\hline & Yes & 60 & 8.9 & 21 & 3.5 & 81 & 6.3 \\
\hline \multirow[t]{2}{*}{ Urine drug test } & Positive & 50 & 7.7 & 14 & 2.4 & 64 & 5.2 \\
\hline & Negative & 600 & 92.3 & 575 & 97.6 & 1175 & 94.8 \\
\hline \multirow[t]{2}{*}{ Ever used tobacco or illicit drugs $* * * *$} & No & 499 & 73.9 & 547 & 90.3 & 1046 & 81.7 \\
\hline & Yes & 176 & 26.1 & 59 & 9.7 & 235 & 18.4 \\
\hline
\end{tabular}

*Missing HIV status for 6 men, 3 women; **missing HSV2 status for 7 men, 4 women; ${ }^{* * *}$ missing PEth for 5 men; ${ }^{* * * *}$ in the study community, tobacco is often smoked with a mixture of other illicit drugs. Prevalence of substance use. HIV-Human Immunodeficiency Virus, HSV2-herpes Simplex Virus. AUDIT- Alcohol Use Disorders Identification Test. SD - standard deviation. 
Overall, 51.4\% of participants reported ever using any alcohol. The overall prevalence of alcohol misuse (AUDIT $\geq 8$ ) was (19.4\%, 95\% CI:17.3-21.7), with males reporting this slightly more frequently (21.5\% in males vs. $17.0 \%$ in females, $p=0.09$, Table 1 . A total of 73 (5.7\%, 95\% CI:4.5-7.2) participants reported an AUDIT score of $\geq 20$ (classified as alcohol dependence; $8.0 \%$ in males and $3.1 \%$ in females). Among all participants, 261 (20.5\%, 95\% CI:18.2-22.7) had whole blood PEth 16:0/18:1 concentrations of $\geq 20 \mathrm{ng} / \mathrm{mL}$ indicating significant drinking ( $24.6 \%$ in males vs. $15.9 \%$ in females, $p<$ 0.001), 55 (4.3\%, 95\% CI:3.3-5.6) had whole blood PEth 16:0/18:1 concentrations of $\geq 210 \mathrm{ng} / \mathrm{mL}$, indicating heavy chronic drinking (5.5\% in males vs. $3.0 \%$ in females, $p=0.03)$, and $81(6.3 \%, 95 \% \mathrm{CI}$ : 5.1-7.8) were classified as binge drinkers in the past month based on TLFB (8.9\% male, 3.5\% female, $p=0.002)$. The overall prevalence of self-reported lifetime use of tobacco or illicit drugs was $(18.4 \%$, 95\% CI: 16.3-20.6); 26.1\% males vs. 9.7\% females, and the overall prevalence of recent illicit drug use based on a urine test was 5.2\% (7.7\% males, $2.4 \%$ females, $p=0.02)$. Among participants with a positive urine drug test, the only drugs detected were cannabinoids $(n=58,90.6 \%)$ and amphetamines $(n=6,9.4 \%)$.

\section{Risk Factors for Substance Use}

Table 2 shows the relationship between examined characteristics and alcohol misuse (AUDIT $\geq$ 8 ) in the study. In univariable analyses, the prevalence of alcohol misuse (AUDIT $\geq 8$ ) was higher in those aged 20-24 years compared to 15-19-year olds (Odds-Ratio (OR) 1.89, 95\% Confidence Interval (CI): 1.37-2.62). AUDIT $\geq 8$ was not associated with gender (either in the full sample or when restricted only to those who reported any drinking), but was crudely associated with all other characteristics examined except marital status, duration of time spent in the fishing community, and whether information on the dangers of alcohol misuse were taught in school. After adjustment for potential confounders, AUDIT $\geq 8$ remained strongly associated with older age, lower education, fishingrelated occupation status, being non-Ugandan, smoking (aOR 4.45, 95\% CI: 2.84-6.97), HSV2 (aOR 1.87, 95\% CI: 1.34-2.59) and depressive symptoms (compared to minimal symptoms, aOR 2.35, 95\% CI: 1.41-3.91 and aOR 2.30, 95\% CI: 0.53-9.90 for mild and moderate-severe, respectively) (Table 2a). After adjustment, HIV was no longer associated with AUDIT $\geq 8$ (aOR 1.50, 95\% CI: 0.72-3.13). 
Table 2. Risk factors for alcohol misuse (AUDIT score $\geq 8$ ) among young people aged 15-24 years in fishing communities in Uganda.

\begin{tabular}{|c|c|c|c|c|c|c|c|}
\hline Characteristic & Categories & $\begin{array}{l}\text { Low drinking } \\
\text { (AUDIT } \leq 7 \text { ) }\end{array}$ & $\begin{array}{l}\text { Heavy drinking } \\
\text { (AUDIT } \geq 8 \text { ) }\end{array}$ & $\begin{array}{l}\text { Crude odds ratio }(95 \% \\
\text { CI) }\end{array}$ & $\begin{array}{c}p- \\
\text { value }\end{array}$ & $\begin{array}{c}\text { Adjusted odds } \\
\text { ratio } \\
(95 \% \mathrm{CI}) *\end{array}$ & $\begin{array}{c}p- \\
\text { value }\end{array}$ \\
\hline & All participants & $1281(80.6)$ & $248(19.4)$ & & & & \\
\hline \multirow[t]{2}{*}{ Gender } & Male & $530(78.5)$ & $145(21.5)$ & 1 (Reference) & & 1 (Reference) & \\
\hline & Female & $503(83.0)$ & $103(17.0)$ & $0.75(0.54-1.05)$ & 0.09 & $0.80(0.57-1.14)$ & 0.20 \\
\hline \multirow[t]{2}{*}{ Age group } & $15-19$ & $421(86.5)$ & $66(13.6)$ & 1 (Reference) & & 1 (Reference) & \\
\hline & $20-24$ & $612(77.1)$ & $182(22.9)$ & $1.89(1.37-2.62)$ & 0.001 & $1.97(1.46-2.67)$ & $<0.001$ \\
\hline \multirow[t]{2}{*}{ Education } & None/primary & $605(77.0)$ & $181(23.0)$ & 1 (Reference) & & 1 (Reference) & \\
\hline & Secondary and above & $428(86.5)$ & $67(13.5)$ & $0.52(0.34-0.799)$ & 0.005 & $0.49(0.33-0.74)$ & 0.002 \\
\hline \multirow[t]{6}{*}{ Tribe } & Central & $390(81.4)$ & 89 (18.6) & 1 (Reference) & & 1 (Reference) & \\
\hline & Eastern & $356(82.8)$ & $74(17.2)$ & $0.91(0.64-1.30)$ & & $0.87(0.62-1.21)$ & \\
\hline & Northern & $27(90.0)$ & $3(10.0)$ & $0.49(0.14-1.73)$ & & $0.48(0.15-1.50)$ & \\
\hline & South-Western & $164(81.6)$ & $37(18.4)$ & $0.99(0.65-1.51)$ & & $0.86(0.56-1.32)$ & \\
\hline & Non-Ugandan & $77(64.7)$ & $42(35.3)$ & $2.39(1.62-3.52)$ & & $2.22(1.48-3.32)$ & \\
\hline & Other & $19(86.4)$ & $42(35.3)$ & $0.69(0.218-2.2)$ & 0.006 & $0.60(0.18-2.00)$ & 0.01 \\
\hline \multirow[t]{3}{*}{ Religion } & Christian & $767(79.4)$ & $199(20.6)$ & 1 (Reference) & & 1 (Reference) & \\
\hline & Muslim & $257(85.4)$ & $44(14.6)$ & $0.66(0.43-1.03)$ & & $0.68(0.43-1.06)$ & \\
\hline & Traditional/other & $9(64.3)$ & $5(35.71)$ & $2.14(0.85-5.38)$ & 0.04 & $1.82(0.64-5.17)$ & 0.10 \\
\hline \multirow[t]{3}{*}{ Parity } & 0 & $574(83.7)$ & $112(16.3)$ & 1 (Reference) & & 1 (Reference) & \\
\hline & 1 & $258(74.6)$ & $88(25.4)$ & $1.75(1.20-2.55)$ & & $1.65(1.05-2.60)$ & \\
\hline & $\geq 2$ & $201(80.7)$ & 48 (19.3) & $1.22(0.82-1.83)$ & 0.02 & $1.22(0.73-2.04)$ & 0.08 \\
\hline \multirow[t]{4}{*}{ Marital status } & Cohabiting & $171(76.3)$ & $53(23.7)$ & 1 (Reference) & & 1 (Reference) & \\
\hline & Married & $340(82.7)$ & $71(17.3)$ & $0.67(0.45-1.02)$ & & $0.57(0.38-0.87)$ & \\
\hline & Separated/divorced/widowed & $65(73.9)$ & $23(26.1)$ & $1.14(0.73-1.79)$ & & $0.83(0.47-1.47)$ & \\
\hline & Single & $457(81.9)$ & $101(18.1)$ & $0.71(0.48-1.07)$ & 0.15 & $0.89(0.57-1.40)$ & 0.10 \\
\hline
\end{tabular}




\begin{tabular}{|c|c|c|c|c|c|c|c|}
\hline \multirow{4}{*}{ Income } & $<200$ & $642(83.6)$ & $126(16.4)$ & 1 (Reference) & & 1 (Reference) & \\
\hline & $200 \mathrm{~K}-299 \mathrm{~K}$ & $162(76.1)$ & $51(23.9)$ & $1.60(1.11-2.32)$ & & $1.05(0.71-1.57)$ & \\
\hline & $300 \mathrm{~K}-399 \mathrm{~K}$ & $121(81.2)$ & $28(18.8)$ & $1.17(0.75-1.86)$ & & $0.85(0.45-1.58)$ & \\
\hline & $\geq 400 \mathrm{~K}$ & $108(71.5)$ & $43(28.5)$ & $2.03(1.36-3.03)$ & 0.002 & $1.35(0.77-2.36)$ & 0.59 \\
\hline \multirow[t]{6}{*}{ Occupation } & Agriculture & $167(82.7)$ & $35(17.3)$ & 1 (Reference) & & 1 (Reference) & \\
\hline & Entertainment & $148(75.1)$ & $49(24.9)$ & $1.58(0.95-2.63)$ & & $1.41(0.82-2.41)$ & \\
\hline & Fishing related & $268(70.2)$ & $114(29.8)$ & $2.03(1.21-3.41)$ & & $2.05(1.18-3.30)$ & \\
\hline & Itinerary trade & $164(87.7)$ & $23(12.3)$ & $0.67(0.36-1.25)$ & & $0.67(0.37-1.23)$ & \\
\hline & Unemployed & $250(91.2)$ & $24(8.8)$ & $0.46(0.27-0.77)$ & & $0.53(0.30-0.90)$ & \\
\hline & Other & $36(92.3)$ & $3(7.7)$ & $0.40(0.06-2.73)$ & $<0.001$ & $0.50(0.08-3.07)$ & $<0.001$ \\
\hline \multirow{4}{*}{$\begin{array}{l}\text { Duration in } \\
\text { community }\end{array}$} & $\leq 6$ months & $453(82.4)$ & 97 (17.64) & 1 (Reference) & & 1 (Reference) & \\
\hline & 7-12 months & $173(80.8)$ & $41(19.2)$ & $1.11(0.79-1.56)$ & & $1.09(0.72-1.63)$ & \\
\hline & $1-4$ years & $311(79.5)$ & $80(20.5)$ & $1.20(0.84-1.71)$ & & $1.00(0.68-1.47)$ & \\
\hline & $\geq 5$ years & $96(76.2)$ & $30(23.8)$ & $1.46(0.89-2.39)$ & 0.52 & $1.10(0.64-1.87)$ & 0.94 \\
\hline \multirow{2}{*}{ Alcohol adverts } & No & $227(86.3)$ & $36(13.7)$ & 1 (Reference) & & 1 (Reference) & \\
\hline & Yes & $806(79.2)$ & $212(20.8)$ & $1.66(1.02-2.70)$ & 0.04 & $1.62(0.95-2.79)$ & 0.08 \\
\hline \multirow{2}{*}{ School curriculum } & No & $333(78.9)$ & 89 (21.1) & 1 (Reference) & & 1 (Reference) & \\
\hline & Yes & $700(81.5)$ & $159(18.51)$ & $0.85(0.61-1.18)$ & 0.31 & $1.02(0.69-1.49)$ & 0.94 \\
\hline \multirow[t]{2}{*}{ Smoking } & No & $923(86.0)$ & $150(14.0)$ & 1 (Reference) & & 1 (Reference) & \\
\hline & Yes & $110(52.9)$ & $98(47.1)$ & $5.48(3.90-7.70)$ & $<0.001$ & $4.45(2.84-6.97)$ & $<0.001$ \\
\hline \multirow[t]{2}{*}{ Illicit drug use } & No & $975(82.4)$ & 209 (17.7) & 1 (Reference) & & 1 (Reference) & \\
\hline & Yes & $27(49.1)$ & $28(50.9)$ & $4.73(3.11-7.20)$ & $<0.001$ & $1.38(0.78-2.43)$ & 0.25 \\
\hline \multirow[t]{2}{*}{ HIV } & Negative & $978(81.2)$ & 227 (18.8) & 1 (Reference) & & 1 (Reference) & \\
\hline & Positive & $47(70.2)$ & $20(29.9)$ & $4.83(3.03-7.72)$ & $<0.001$ & $1.50(0.72-3.13)$ & 0.27 \\
\hline \multirow[t]{2}{*}{ HSV2 } & Negative & $728(84.3)$ & 136 (15.7) & 1 (Reference) & & 1 (Reference) & \\
\hline & Positive & $295(72.7)$ & $111(27.3)$ & $2.01(1.55-2.61)$ & $<0.001$ & $1.87(1.34-2.59)$ & $<0.001$ \\
\hline
\end{tabular}




\begin{tabular}{|c|c|c|c|c|c|c|c|}
\hline \multirow{3}{*}{$\begin{array}{l}\text { Depressive } \\
\text { symptoms }\end{array}$} & Minimal & $931(82.6)$ & $196(17.4)$ & 1 (Reference) & & 1 (Reference) & \\
\hline & Mild & $82(65.6)$ & $43(34.4)$ & $2.49(1.43-4.34)$ & & $2.35(1.41-3.91)$ & \\
\hline & Moderate-severe & $15(68.2)$ & $7(31.8)$ & $2.22(1.10-4.44)$ & 0.01 & $2.30(0.53-9.90)$ & 0.01 \\
\hline
\end{tabular}

${ }^{*}$ All adjusted ORs controlled for age, gender, education, tribe and religion; adjusted ORs for parity, marital status, income, occupation, duration in community, alcohol adverts and school curriculum additionally controlled for each other; adjusted ORs for smoking, alcohol use, depressive symptoms, HIV and HSV2 additionally adjusted for each other (with the exception of HIV and HSV2, which were not controlled for each other). CI-confidence interval. 
Table 3 shows the relationship between the examined characteristics and PEth 16:0/18:1 $\geq$ $20 \mathrm{ng} / \mathrm{mL}$ (representing heavy drinking). In univariable analysis, all characteristics were associated with PEth 16:0/18:1 $\geq 20 \mathrm{ng} / \mathrm{mL}$ except for duration of time lived in the community and exposure to alcohol adverts. In multivariable analyses, alcohol misuse according to PEth remained strongly associated with male gender, older age, low education, being non-Ugandan, being non-Muslim, occupations in itinerant trade, fish-related or entertainment industries, parity, smoking (aOR 3.16, 95\% CI: 1.76-5.68), illicit drug use (aOR 2.72, 95\% CI: 1.29-5.74) and HSV2 (aOR 1.87, 95\% CI: 1.362.56). After adjustment, HIV was no longer associated with PEth 16:0/18:1 $\geq 20 \mathrm{ng} / \mathrm{mL}$ (aOR 1.43, 95\% CI: 0.93-2.19). 
Table 3. Risk factors for alcohol misuse (PEth 16:0/18:1 $\geq 20 \mathrm{ng} / \mathrm{mL}$ ) among young people aged 15-24 years in fishing communities in Uganda.

\begin{tabular}{|c|c|c|c|c|c|c|c|}
\hline Characteristic & Categories & $\begin{array}{c}\text { Not detected } \\
\text { (PEth 16:0/18:1 }<20 \\
\text { ng/mL) } \\
\text { N(\%) }\end{array}$ & $\begin{array}{c}\text { Heavy drinking } \\
\text { (PEth 16:0/18:1 } \geq 20 \\
\text { ng/mL) } \\
\mathrm{N}(\%)\end{array}$ & $\begin{array}{c}\text { Crude odds } \\
\text { ratio } \\
(95 \% \mathrm{CI})\end{array}$ & $\begin{array}{c}p- \\
\text { value }\end{array}$ & $\begin{array}{l}\text { Adjusted odds } \\
\text { ratio( } 95 \% \mathrm{CI})\end{array}$ & $p$-value \\
\hline & All participants & 1015 (79.5) & $261(20.5)$ & & & & \\
\hline \multirow[t]{2}{*}{ Gender } & Male & $505(75.4)$ & $165(24.6)$ & 1 (Reference) & & 1 (Reference) & \\
\hline & Female & $510(84.2)$ & $96(15.8)$ & $0.58(0.44-0.76)$ & $<0.001$ & $0.58(0.42-0.81)$ & 0.003 \\
\hline \multirow[t]{2}{*}{ Age } & $15-19$ & $440(90.4)$ & $47(9.7)$ & 1 (Reference) & & 1 (Reference) & \\
\hline & $20-24$ & $575(72.9)$ & $214(27.1)$ & $3.48(2.28-5.31)$ & $<0.001$ & $3.72(2.45-5.63)$ & $<0.001$ \\
\hline \multirow[t]{2}{*}{ Education } & None/primary & & $189(24.1)$ & 1 (Reference) & & 1 (Reference) & \\
\hline & $\begin{array}{c}\text { Secondary and } \\
\text { above }\end{array}$ & $420(85.4)$ & $72(14.6)$ & $0.54(0.40-0.72)$ & $<0.001$ & $0.48(0.36-0.65)$ & $<0.001$ \\
\hline \multirow[t]{6}{*}{ Tribe } & Central & $374(78.7)$ & $101(21.3)$ & 1 (Reference) & & 1 (Reference) & \\
\hline & Eastern & $358(83.5)$ & $71(16.6)$ & $0.73(0.53-1.02)$ & & $0.70(0.49-1.00)$ & \\
\hline & Northern & $27(90.0)$ & $3(10.0)$ & $0.41(0.15-1.12)$ & & $0.42(0.18-0.99)$ & \\
\hline & South-Western & $154(76.6)$ & $47(23.4)$ & $1.13(0.68-1.89)$ & & $0.96(0.57-1.62)$ & \\
\hline & Non-Ugandan & $82(68.9)$ & $37(31.1)$ & $1.67(1.06-2.63)$ & & $1.50(0.93-2.41)$ & \\
\hline & Other & $20(90.9)$ & $2(9.1)$ & $0.30(0.09-1.49)$ & 0.02 & $0.30(0.07-1.37)$ & 0.04 \\
\hline \multirow[t]{3}{*}{ Religion } & Christian & $751(78.1)$ & $211(21.9)$ & 1 (Reference) & & 1 (Reference) & \\
\hline & Muslim & $256(85.3)$ & $44(14.7)$ & $0.61(0.47-0.80)$ & & $0.63(0.49-0.81)$ & \\
\hline & Traditional/Other & $8(57.1)$ & $6(42.9)$ & $2.27(0.96-7.41)$ & 0.001 & $2.05(0.68-6.17)$ & 0.01 \\
\hline \multirow[t]{3}{*}{ Parity } & 0 & $571(83.5)$ & $113(16.5)$ & 1 (Reference) & & 1 (Reference) & \\
\hline & 1 & $249(72.2)$ & $96(27.8)$ & $1.95(1.32-2.87)$ & & $1.70(1.06-2.73)$ & \\
\hline & $\geq 2$ & $195(79)$ & $52(21.1)$ & $1.35(0.84-2.16)$ & 0.008 & $1.22(0.66-2.36)$ & 0.04 \\
\hline \multirow[t]{2}{*}{ Marital status } & Cohabiting & $170(76.2)$ & $53(23.8)$ & 1 (Reference) & & 1 (Reference) & \\
\hline & Married & $330(80.9)$ & 78 (19.1) & $0.76(0.53-1.09)$ & & $0.60(0.40-0.92)$ & \\
\hline
\end{tabular}




\begin{tabular}{|c|c|c|c|c|c|c|c|}
\hline & $\begin{array}{c}\text { Separated/divorced/ } \\
\text { widowed }\end{array}$ & $60(68.2)$ & $28(31.8)$ & $1.50(0.71-3.14)$ & & $1.02(0.41-2.54)$ & \\
\hline & Single & $455(81.7)$ & $102(18.3)$ & $0.72(0.53-0.97)$ & 0.04 & $0.93(0.60-1.47)$ & 0.13 \\
\hline \multirow[t]{4}{*}{ Income } & $<200$ & $644(84)$ & $123(16)$ & 1 (Reference) & & 1 (Reference) & \\
\hline & $200-299 \mathrm{~K}$ & $146(68.9)$ & $66(31.1)$ & $2.37(1.63-3.44)$ & & $1.35(0.81-2.25)$ & \\
\hline & $300-399 \mathrm{~K}$ & $114(77.0)$ & $34(23.0)$ & 1.56 (1.07-2.27) & & $0.59(0.60-1.51)$ & \\
\hline & $\geq 400 \mathrm{~K}$ & $111(74.5)$ & $38(25.5)$ & 1.79 (1.15-2.79) & 0.002 & $0.92(0.51-1.67)$ & 0.25 \\
\hline \multirow[t]{6}{*}{ Occupation } & Agriculture & $176(87.1)$ & $26(12.9)$ & 1 (Reference) & & 1 (Reference) & \\
\hline & Entertainment & $152(77.2)$ & $45(22.8)$ & $2(1.22-3.3)$ & & $2.06(1.19-3.54)$ & \\
\hline & Fishing related & $250(66.3)$ & $127(33.7)$ & $3.34(2.24-5.05)$ & & $3.01(1.81-5.00)$ & \\
\hline & Itinerary trade & $150(80.2)$ & 37 (19.8) & $1.67(1.08-2.58)$ & & $1.90(1.20-3.05)$ & \\
\hline & Unemployed & $252(92.0)$ & $22(8.0)$ & $0.59(0.30-1.15)$ & & $0.94(0.43-2.09)$ & \\
\hline & Other & $35(89.7)$ & $4(10.3)$ & $0.77(0.22-2.71)$ & $<0.001$ & $0.93(0.29-2.94)$ & 0.004 \\
\hline \multirow{4}{*}{$\begin{array}{l}\text { Duration in } \\
\text { community }\end{array}$} & $\leq 6$ months & $444(80.9)$ & $105(19.1)$ & 1 (Reference) & & 1 (Reference) & \\
\hline & 7 months to 1 year & $169(79.3)$ & $44(20.7)$ & $1.10(0.77-1.57)$ & & $1.00(0.67-1.47)$ & \\
\hline & $1-4$ years & $303(78.1)$ & $85(21.9)$ & 1.19 (0.93-1.52) & & $0.88(0.61-1.26)$ & \\
\hline & $\geq 5$ years & 99 (78.6) & $27(21.4)$ & $1.15(0.68-2.00)$ & 0.53 & $0.70(0.38-1.30)$ & 0.63 \\
\hline \multirow{2}{*}{ Alcohol adverts } & No & $224(85.5)$ & 38 (14.5) & 1 (Reference) & & 1 (Reference) & \\
\hline & Yes & $791(78.0)$ & $223(22.0)$ & $1.66(0.95-2.90)$ & 0.07 & $1.69(0.97-3.00)$ & 0.07 \\
\hline \multirow{2}{*}{ School curriculum } & No & $318(75.4)$ & $104(24.6)$ & 1 (Reference) & & 1 (Reference) & \\
\hline & Yes & $697(81.6)$ & $157(18.4)$ & $0.69(0.50-0.95)$ & 0.02 & $0.76(0.52-1.10)$ & 0.14 \\
\hline \multirow[t]{2}{*}{ Smoking } & No & $902(84.4)$ & $167(15.6)$ & 1 (Reference) & & 1 (Reference) & \\
\hline & Yes & $113(54.6)$ & $94(45.4)$ & $4.49(3.19-6.33)$ & $<0.001$ & $3.16(1.76-5.68)$ & $<0.001$ \\
\hline \multirow[t]{2}{*}{ Illicit drug use } & No & $964(81.6)$ & $218(18.4)$ & 1 (Reference) & & 1 (Reference) & \\
\hline & Yes & $21(38.2)$ & $34(61.8)$ & $7.03(4.25-11.64)$ & $<0.001$ & $2.72(1.29-5.74)$ & 0.01 \\
\hline \multirow[t]{2}{*}{ HIV } & Negative & $968(80.6)$ & $233(91.4)$ & 1 (Reference) & & 1 (Reference) & \\
\hline & Positive & $45(67.2)$ & $22(32.8)$ & $2.03(1.5-2.75)$ & $<0.001$ & $1.43(0.93-2.19)$ & 0.10 \\
\hline
\end{tabular}




\begin{tabular}{|c|c|c|c|c|c|c|c|}
\hline \multirow[t]{2}{*}{ HSV2 } & Negative & $721(83.6)$ & $141(16.4)$ & 1 (Reference) & & 1 (Reference) & \\
\hline & Positive & 291(71.9) & $114(28.2)$ & $2.22(1.59-2.52)$ & $<0.001$ & $1.87(1.36-2.56)$ & $<0.001$ \\
\hline \multirow{3}{*}{ Depressive symptoms } & Minimal & 907 (80.8) & $216(19.2)$ & 1 (Reference) & & 1 (Reference) & \\
\hline & Mild & $86(69.4)$ & $38(30.7)$ & $1.85(1.08-3.2)$ & & $1.75(0.94-3.26)$ & \\
\hline & Moderate-severe & $22(75.9)$ & $7(24.1)$ & $1.33(0.74-2.4)$ & 0.02 & $1.26(0.45-3.50)$ & 0.15 \\
\hline
\end{tabular}


Supplementary Table 1 shows that factors associated with hazardous drinking (PEth 16:0/18:1 $\geq 210 \mathrm{ng} / \mathrm{mL}$ ) at multivariable analysis included male gender, older age group 20-24 and low education.

Table 4 shows the relationship between the characteristics and binge drinking in the past month ascertained using TLFB. In crude analysis, binge drinking was associated with all characteristics except tribe, occupation, duration in community, exposure to school curriculum on alcohol, and depressive symptoms. After adjusting for potential confounders, binge drinking was associated with older age, low education, being single, parity, exposure to alcohol adverts, and smoking, illicit drug use and HSV2, with weak evidence for association with mild depressive symptoms. 
Table 4. Risk factors for alcohol misuse (binge drinking in previous month) among young people aged 15-24 years in fishing communities in Uganda

\begin{tabular}{|c|c|c|c|c|c|c|}
\hline Characteristics & Categories & $\begin{array}{c}\text { Binge drinking } \\
\mathrm{N}(\%)\end{array}$ & Crude odds ratio $(95 \% \mathrm{CI})$ & $p$-value & $\begin{array}{c}\text { Adjusted odds ratio } \\
(95 \% \mathrm{CI}) *\end{array}$ & $p$-value \\
\hline & All participants & $81(6.3)$ & & & & \\
\hline \multirow[t]{2}{*}{ Gender } & Male & $60(8.9)$ & 1 (Reference) & & 1 (Reference) & \\
\hline & Female & $21(3.5)$ & $0.37(0.20-0.67)$ & 0.002 & $0.39(0.22-0.70$ & 0.03 \\
\hline \multirow[t]{2}{*}{ Age group } & $15-19$ & $22(4.5)$ & 1 (Reference) & & 1 (Reference) & \\
\hline & $20-24$ & $59(7.4)$ & $1.70(0.99-2.92)$ & 0.06 & $1.77(1.02-3.08)$ & 0.05 \\
\hline \multirow[t]{2}{*}{ Education } & None/primary & $62(7.9)$ & 1 (Reference) & & 1 (Reference) & \\
\hline & Secondary and above & $19(3.8)$ & $0.47(0.22-1.00)$ & 0.05 & $0.46(0.21-0.97)$ & 0.04 \\
\hline \multirow[t]{6}{*}{ Tribe } & Central & $30(6.3)$ & 1 (Reference) & & 1 (Reference) & \\
\hline & Eastern & $18(4.2)$ & $0.65(0.29-1.46)$ & & $0.63(0.29-1.38)$ & \\
\hline & Northern & $2(6.7)$ & $1.07(0.26-4.49)$ & & $1.12(0.31-4.05)$ & \\
\hline & South-Western & $15(7.5)$ & $1.21(0.50-2.91)$ & & $1.08(0.46-2.54)$ & \\
\hline & Non-Ugandan & $14(11.8)$ & $2.00(1.08-3.38)$ & & $1.69(0.90-3.18)$ & \\
\hline & Other & $2(9.1)$ & $1.50(0.33-0.12)$ & 0.31 & $1.21(0.27-5.41)$ & 0.48 \\
\hline \multirow[t]{3}{*}{ Religion } & Christian & $68(7.0)$ & 1 (Reference) & & 1 (Reference) & \\
\hline & Muslim & $11(3.7)$ & $0.50(0.28-0.89)$ & & $0.55(0.31-0.98)$ & \\
\hline & Traditional/other & $2(14.3)$ & $2.20(0.50-9.73)$ & 0.02 & $1.42(0.34-6.02)$ & 0.09 \\
\hline \multirow[t]{3}{*}{ Parity } & 0 & $34(5.0)$ & 1 (Reference) & & 1 (Reference) & \\
\hline & 1 & $33(9.5)$ & $2.02(1.28-3.19)$ & & $2.77(1.59-4.83)$ & \\
\hline & $\geq 2$ & $14(5.6)$ & $1.14(0.56-2.32)$ & $<0.001$ & $2.02(0.93-4.39)$ & 0.00 \\
\hline \multirow[t]{4}{*}{ Marital status } & Cohabiting & $15(6.7)$ & 1 (Reference) & & 1 (Reference) & \\
\hline & Married & $23(5.6)$ & $0.83(0.49-1.40)$ & & $0.59(0.32-1.09)$ & \\
\hline & Separated/divorced/widowed & $7(8.0)$ & $1.20(0.31-4.63)$ & & $0.84(0.24-2.87)$ & \\
\hline & Single & $36(6.5)$ & $0.96(0.43-2.13)$ & 0.43 & $1.10(0.38-3.17)$ & 0.01 \\
\hline
\end{tabular}




\begin{tabular}{|c|c|c|c|c|c|c|}
\hline \multirow[t]{4}{*}{ Income } & $<200$ & $34(4.4)$ & 1 (Reference) & & 1 (Reference) & \\
\hline & $200 k-300 k$ & $23(10.8)$ & $2.61(1.39-4.91)$ & & $1.59(0.76-3.30)$ & \\
\hline & $300 k-400 k$ & $11(7.4)$ & $1.72(0.89-3.34)$ & & $1.12(0.51-2.48)$ & \\
\hline & $\geq 400 \mathrm{~K}$ & $13(8.6)$ & $2.03(1.05-3.93)$ & 0.02 & $1.11(0.51-2.42)$ & 0.51 \\
\hline \multirow[t]{6}{*}{ Occupation } & Agriculture & $12(5.9)$ & 1 (Reference) & & 1 (Reference) & \\
\hline & Entertainment & $7(3.6)$ & $0.58(0.19-1.82)$ & & $0.66(0.24-1.85)$ & \\
\hline & Fishing related & $45(11.8)$ & $2.11(0.83-5.37)$ & & $1.60(0.54-4.73)$ & \\
\hline & Itinerary trade & $5(2.7)$ & $0.44(0.13-1.45)$ & & $0.50(0.14-1.82)$ & \\
\hline & Unemployed & $8(2.9)$ & $0.48(0.22-1.02)$ & & $0.74(0.35-1.54)$ & \\
\hline & Other & $4(10.3)$ & $1.81(0.41-7.94)$ & 0.07 & $2.40(0.49-11.71)$ & 0.34 \\
\hline \multirow[t]{4}{*}{ Duration in community } & $\leq 6$ months & $28(5.1)$ & 1 (Reference) & & 1 (Reference) & \\
\hline & 7 months to 1 year & $10(4.7)$ & $0.91(0.45-1.87)$ & & $0.71(0.34-1.47)$ & \\
\hline & $1-4$ years & $28(7.2)$ & $1.44(0.88-2.35)$ & & $0.96(0.56-1.63))$ & \\
\hline & $\geq 4$ years & $15(11.9)$ & $2.52(1.28-4.95)$ & 0.09 & $1.55(0.73-3.28)$ & 0.29 \\
\hline \multirow[t]{2}{*}{ Alcohol adverts } & No & $9(3.4)$ & 1 (Reference) & & 1 (Reference) & \\
\hline & Yes & $72(7.1)$ & $2.15(1.37-3.38)$ & 0.002 & $2.09(1.17-3.73)$ & 0.02 \\
\hline \multirow[t]{2}{*}{ School curriculum } & No & $31(7.4)$ & 1 (Reference) & & 1 (Reference) & \\
\hline & Yes & $50(5.8)$ & $0.78(0.42-1.43)$ & 0.40 & $0.90(0.51-1.62)$ & 0.72 \\
\hline \multirow[t]{2}{*}{ Smoking } & No & $47(4.4)$ & 1 (Reference) & & 1 (Reference) & \\
\hline & Yes & $34(16.4)$ & $4.27(2.52-7.22)$ & $<0.001$ & $2.34(1.28-4.27)$ & 0.01 \\
\hline \multirow[t]{2}{*}{ Illicit drug use } & No & $59(5.0)$ & 1 (Reference) & & 1 (Reference) & \\
\hline & Yes & $17(26.6)$ & $6.84(3.97-11.80)$ & $<0.001$ & $2.54(1.00-6.41)$ & 0.05 \\
\hline \multirow[t]{2}{*}{ HIV } & Negative & $72(6.0)$ & 1 (Reference) & & 1 (Reference) & \\
\hline & Positive & $9(13.4)$ & $2.44(1.06-5.64)$ & $<0.001$ & $2.97(0.82-10.73)$ & 0.09 \\
\hline \multirow[t]{2}{*}{ HSV2 } & Negative & $372(31.3)$ & 1 (Reference) & & 1 (Reference) & \\
\hline & Positive & $34(42.0)$ & $1.59(0.97-2.60)$ & 0.06 & $1.77(1.03-3.04)$ & 0.04 \\
\hline
\end{tabular}




\begin{tabular}{lccccc}
\hline Depressive symptoms & Minimal & $64(5.7)$ & 1 (Reference) & 1 (Reference) & $2.25(1.14-4.45)$ \\
& Mild & $14(11.2)$ & $2.09(1.13-3.89)$ & 0.06 & $2.64(0.28-25.40)$ \\
& Moderate-severe & $3(10.3)$ & $1.92(0.60-6.09)$ & 0.05 \\
\hline
\end{tabular}

*All adjusted ORs controlled for age, gender, education, tribe and religion; adjusted ORs for parity, marital status, income, occupation, duration in community, alcohol adverts and school curriculum additionally controlled for each other; adjusted ORs for smoking, illicit drug use, depressive symptoms, HIV and HSV2 additionally adjusted for each other (with the exception of HIV and HSV2, which were not controlled for each other). 
Table 5 shows the relationship between the examined characteristics and illicit drug use (positive urine test) in adolescents. In univariable analysis, the prevalence of illicit drug use was associated with gender, age, education, income, occupation, smoking and depressive symptoms. After adjustment for potential confounders, illicit drug use remained positively associated with male gender, older age group, lower education, being single, smoking, and moderate to severe depressive symptoms. 
Table 5. Risk factors for illicit drug use among young people aged 15-24 years in fishing communities in Uganda.

\begin{tabular}{|c|c|c|c|c|c|c|}
\hline Characteristics & Categories & Positive urine drug test & $\begin{array}{c}\text { Crude odds ratio } \\
(95 \% \mathrm{CI})\end{array}$ & $p$-value & Adjusted odds ratio $(95 \% \mathrm{CI})^{*}$ & $p$-value \\
\hline \multirow{3}{*}{ Gender } & All participants & $55(4.4)$ & & & & \\
\hline & Male & $50(7.7)$ & 1 (Reference) & & 1 (Reference) & \\
\hline & Female & $14(2.4)$ & $0.36(0.16-0.81)$ & 0.02 & $0.31(0.13-0.74)$ & 0.01 \\
\hline \multirow{2}{*}{ Age group } & $15-19$ & $13(2.7)$ & 1 (Reference) & & 1 (Reference) & \\
\hline & $20-24$ & $42(5.5)$ & $2.11(1.34-3.34))$ & 0.003 & $2.32(1.42-3.78)$ & $<0.001$ \\
\hline \multirow[t]{2}{*}{ Education } & None/primary & $52(6.9)$ & & & & \\
\hline & Secondary and above & $12(2.5)$ & $0.35(0.19-0.64)$ & 0.002 & $0.39(0.21-0.7)$ & $<0.001$ \\
\hline \multirow[t]{6}{*}{ Tribe } & Central & $19(4.1)$ & 1 (Reference) & & 1 (Reference) & \\
\hline & Eastern & $18(4.4)$ & $1.06(0.50-2.27)$ & & $1.00(0.43-2.11)$ & \\
\hline & Northern & $1(3.3)$ & $0.81(0.09-7.61)$ & & $0.84(0.09-7.71)$ & \\
\hline & South-Western & $12(6.1)$ & $1.53(0.67-3.51)$ & & $1.35(0.58-3.15)$ & \\
\hline & Non-Ugandan & $12(10.5)$ & $2.76(1.42-5.37)$ & & $2.42(1.18-5.00)$ & \\
\hline & Other & $2(9.5)$ & $2.46(0.29-20.97)$ & 0.14 & $2.06(0.18-24.16)$ & 0.28 \\
\hline \multirow[t]{3}{*}{ Religion } & Christian & $48(5.1)$ & 1 (Reference) & & & \\
\hline & Muslim & $15(5.2)$ & $1.03(0.62-1.71)$ & & $1.14(0.68-1.92)$ & \\
\hline & Traditional/other & $1(7.7)$ & $1.55(0.17-14.5)$ & 0.91 & $0.87(0.07-10.62)$ & 0.87 \\
\hline \multirow[t]{3}{*}{ Parity } & 0 & $31(4.6)$ & 1 (Reference) & & 1 (Reference) & \\
\hline & 1 & $23(7.0)$ & $1.54(0.90-2.65)$ & & $2.07(0.92-4.64)$ & \\
\hline & $\geq 2$ & $10(4.18)$ & $0.90(0.44-1.83)$ & 0.13 & $1.58(0.63-3.94)$ & 0.21 \\
\hline \multirow[t]{4}{*}{ Marital status } & Cohabiting & $5(2.4)$ & 1 (Reference) & & 1 (Reference) & \\
\hline & Married & $17(4.3)$ & $1.85(0.83-4.11)$ & & $1.55(0.71-3.38)$ & \\
\hline & Separated/divorced/widowed & $7(8.4)$ & $3.83(1.15-12.80)$ & & $2.94(0.80-10.81)$ & \\
\hline & Single & $35(6.4)$ & $2.86(1.25-6.65)$ & 0.11 & $3.69(1.83-7.47)$ & 0.01 \\
\hline \multirow[t]{4}{*}{ Income } & $<200$ & $20(2.7)$ & 1 (Reference) & & 1 (Reference) & \\
\hline & $200 \mathrm{k}-300 \mathrm{k}$ & $19(9.4)$ & $3.7(1.2-11.97)$ & & $2.37(0.68-8.28)$ & \\
\hline & $300 \mathrm{k}-400 \mathrm{k}$ & $8(5.6)$ & $2.2(0.86-5.39)$ & & $1.11(0.35-3.50)$ & \\
\hline & $\geq 400 \mathrm{~K}$ & $8(5.8)$ & $2.2(1.12-4.528)$ & 0.007 & $1.91(0.75-4.91)$ & 0.46 \\
\hline \multirow[t]{4}{*}{ Occupation } & Agriculture & $9(4.5)$ & 1 (Reference) & & 1 (Reference) & \\
\hline & Entertainment & $6(3.1)$ & $0.68(0.27-1.74)$ & & $0.91(0.38-2.16)$ & \\
\hline & Fishing related & 39 (10.7) & $2.53(1.10-5.80)$ & & $1.51(0.56-4.08)$ & \\
\hline & Itinerary trade & $5(2.8)$ & $0.60(0.22-1.66)$ & & $0.72(0.25-2.12)$ & \\
\hline
\end{tabular}


Unemployed

Other

Duration in community

$$
\leq 6 \text { months }
$$

7 months to 1 year

1-4 years

$\geq 5$ years

Alcohol adverts

No

Yes

No

Yes

No

Yes

Negative

Positive

Negative

positive
$5(1.9)$

0

18 (3.4)

17 (8.3)

$20(5.3)$

$9(7.2)$

9 (3.6)

55 (5.6)

19 (4.7)

45 (5.4)

18 (1.7)

46 (23.1)

$52(4.4)$

3 (4.8)

40 (62.5)

24 (37.5)

Minimal

Mild

Moderate-severe
$0.41(0.14-1.21)$

0.04

$0.81(0.26-2.59)$

0.69

1 (Reference)

$2.59(1.20-5.30)$

$1.61(0.60-3.00)$

$2.22(0.43-5.44)$

0.08

$2.12(1.11-4.06)$

$1.12(0.52-2.41)$

1.35 (0.37-4.90)

0.10

1 (Reference)

$1.51(.73-3.12)$

$1.60(0.87-2.95)$

0.12

1 (Reference)

1.64 (0.91-2.95)

1.15 (0.67-1.98)

0.59

1 (Reference)

13.05 (5.84-29.18)

1 (Reference)

1.22 (0.19-8.01)

1 (Reference)

1.37 (0.81-2.30)

1 (Reference)

$2.22(0.83-5.91)$

$8.23(1.82-37.16)$

*All adjusted ORs controlled for age, gender, education, tribe and religion; adjusted ORs for parity, marital status, income, occupation, duration in community, alcohol adverts and school curriculum additionally controlled for each other; adjusted ORs for smoking, alcohol misuse, depressive symptoms, HIV and HSV2 additionally adjusted for each other (with the exception of HIV and HSV2, which were not controlled for each other). 


\section{Discussion}

Alcohol misuse was common among young people aged 15-24 years in fishing communities in Uganda, and was consistently associated with older age, male gender (except for AUDIT $\geq 8$ ), lower education, smoking, HSV2 and depressive symptoms, regardless of the alcohol use assessment tool used. The prevalence of illicit drug use was lower than that of alcohol misuse. The only drugs detected were cannabinoids and amphetamines. Illicit drug use was associated with male gender, older age, lower education, being single, smoking, and depressive symptoms.

There are limited data on alcohol misuse and illicit drug use from similar settings (8). The prevalence of alcohol misuse in our study was comparable to findings in a recent study among young people in a general population setting in Northern Tanzania, with $11 \%-28 \%$ of males screening positive for alcohol use disorder (AUD)[35], and findings among young people in Goa, India (17.7\%) [36]. However, the prevalence of AUD in our study was much lower than the prevalence $(53 \%$, measured by CAGE) among 14-24 year old female sex workers in Kampala [37], as well as in another study of a key population where, among 1476 young men who reported sex with men in Kenya, $44 \%$ had reported AUD via the AUDIT questionnaire [38]. The prevalence of binge drinking in our study was slightly lower than that reported among young people in the WHO Africa region comprising 47 countries [1). Young men were more likely to screen positive for both AUD based on PEth and binge drinking, and this is consistent with estimates from Africa and other WHO regions (1), although interestingly, AUDIT $\geq 8$ was not associated with gender in this setting. Taken together, these studies further highlight the need for integration and prioritisation of alcohol reduction in HIV prevention interventions among key populations.

The prevalence of illicit drug use was $4.4 \%$, similar to a large population-based survey in South Africa among people aged 15 and older [39]. The most commonly detected drug was cannabis, and this might be attributed to better availability and social acceptability, or lower cost compared to other illicit drugs.

Globally, predictors of harmful alcohol use and illicit drug use among young people include male gender, peer pressure, family experience, psychological factors/mental health, positive expectations regarding alcohol use, violence, socio-economic factors, and increased alcohol and illicit drug availability and marketing [35,40,41]. Furthermore, young people are potentially more likely to engage in reckless behaviour while drunk [42] or whilst on drugs than older adults. In our study, socio-demographic characteristics associated with AUD were similar to those found in studies conducted in more general population settings elsewhere in East Africa and Europe/US. They included male gender, older age group (20-24 years), using illicit drugs and HSV2 infection [37]. However, other studies among female sex workers reported contrary risk factors such as younger age [37]. The findings emphasize the need for integration of alcohol and illicit drug use prevention interventions, and for interventions that will be able to reach males.

Extensive evidence shows that alcohol misuse and illicit drug use are associated with higher HIV risk at societal, community and individual levels' stemming mainly from associations with high risk sexual behaviour, increased HIV shedding and inflammation at mucosal sites [43] and needle sharing for injection drug users [44]. Among people living with HIV, alcohol and illicit drug use may adversely affect disease progression through several mechanisms, including reduced uptake of HIV prevention and care services [45], and through impacts on viral replication, host immunity and treatment compliance and efficacy [43]. Our cross-sectional study did not show a significant association between HIV and AUD or illicit drug use after adjustment for potential confounders, although adjusted odds ratios were greater than 1 for all measures of AUD, and lower limits of $95 \%$ confidence intervals only marginally overlapped 1 . The lack of significant relationships between these variables may be in part due to the relatively small number of HIV events and consequent limited power to detect an association. However, they should not be interpreted as evidence for the absence of such relationships. There was a strong association between substance use and HSV2. Evidence suggests a direct and reciprocal biological interaction between HIV and HSV2 [46]. Additionally, HIV and HSV2 are associated with similar risk factors [age, sex, partner change, 
condom use and male circumcision) [46]. Thus, HSV2 may be considered a proxy measure for risky sexual behaviour. Therefore, prioritizing alcohol interventions and harm reduction among young people in key population settings is critical not only to reducing substance-use-related harms but also to reducing sexually transmitted infections and could accelerate progress towards the UNAIDS 9595-95 HIV epidemic control targets. Our study documents both under-age alcohol use and misuse (the legal age for drinking in Uganda is 18). This implies that additional research to understand the drivers of substance use among minors and interventions to address them is critical.

\section{Strengths and Limitations}

A strength of the study was the large sample size, which meant that we had good power to detect associations with alcohol misuse for most covariates of interest. We selected a populationbased sample and the response rate was high; thus, our findings may be generalisable to similar fishing community settings in the Eastern and Southern Africa regions, although we did not collect detailed information on those who did not participate to further assess this. Another strength is that we used a range of standard tools to assess alcohol use, encompassing the different types of alcohol consumption measures, i.e., tools that report alcohol consumption status, average volume alcohol consumption, and frequency and volume of binge drinking. We identified several key covariates that were strongly associated with alcohol use across all three measures used. These standard tools are essential for monitoring public health and evaluating alcohol control policies and other interventions.

Our results might be less prone to social desirability bias/misreporting, which could bias our estimates of association either towards or away from the null, for two reasons: (i) use of objective measures to supplement self-reported measures of alcohol and illicit drug use and sexual risk behaviour; (ii) administration of ACASI, which was acceptable and feasible. Previous literature suggests that ACASI allows more accurate reporting of sensitive behaviours including alcohol (mis)use. However, we did not have an interviewer-administered comparison group to test this hypothesis in our study. Therefore, our findings might not be generalizable to AUDIT being administered face-to-face. We further minimised reporting bias by using young gender-matched interviewers, and by using a customised TLFB calendar and pictorial displays of standard alcoholic drinks [35]. Our survey had a response rate (based on residents listed in a previous census) of approximately $80 \%$, therefore we cannot exclude the possibility that our results are subject to selection bias. Non-response, especially in a study of a controversial topic such as substance abuse or sexual behaviour, could potentially introduce selection bias and consequently lead to underestimation of outcome and risk factor prevalence [47], and may affect the internal validity of the study [48]. Interpretation of the effect sizes found in this study should be undertaken with caution, as these may depend on the specific characteristics of the samples included in our study. A further limitation of our study was the cross-sectional design, which precludes us from demonstrating causality between independent variables and AUD and or illicit drug use, and between AUD and HIV or HSV2. We chose to model HIV and HSV2 as risk factors for alcohol misuse and illicit drug use since alcohol misuse and illicit drug use were our primary outcomes of interest, but reverse causation is a plausible explanation for the associations seen.

\section{Conclusions}

Alcohol misuse and illicit drug use are common among young people in fishing communities in Uganda and associated with sexual risk behaviour and smoking. Uganda is an HIV-hyperendemic country, with one of the youngest and fastest-growing populations and one of the highest estimated alcohol per capita consumption rates globally [1]. There is potential for additional benefit of combined prevention and intervention activities targeting alcohol and illicit drug use, and HIV particularly, in key population settings.

Supplementary Materials: The following are available online at www.mdpi.com/1660-4601/17/7/2401/s1, Table T1: Risk factors for alcohol misuse (PEth 16:0/18:1 $\geq 210 \mathrm{ng} / \mathrm{mL}$ ) among young people aged 15-24 years in fishing communities in Uganda. 
Author contributions: Conceptualisation: M.K.O., E.L.W., S.C., H.A.W. and J.S; Methodology: M.K.O., E.L.W., S.C., H.A.W., J.S.; Software: M.K.O.; Validation: M.K.O., J.S.N., P.N., M.S. and R.K.; Formal analysis: M.K.O., E.L.W. and J.S.; Investigation: M.K.O., J.S.N., P.N., M.S, and R.K.; Resources: M.K.O., J.S.N., P.N., M.S., and R.K.; Data curation: M.K.O., E.L.W., J.S. and J.S.N.; Writing-original draft preparation: M.K.O. and E.L.W.; Writing-review and editing: M.K.O., E.L.W., S.C. and H.A.W.; Visualisation: M.K.O., J.S. and E.L.W.; Supervision: M.K.O., J.S. and E.L.W.; Project administration: M.K.O.; Funding acquisition: M.K.O., E.L.W. and J.S.; Final approval of the article: All co-authors. All authors have read and agreed to the published version of the manuscript.

Funding: This research was funded by the UK Medical Research Council (MRC) and the UK Department for International Development (DFID) under the MRC/DFID Concordat agreement, the MRC Tropical Epidemiology Group (TEG) (MR/K012126/1) and International AIDS Vaccine Initiative (made possible by the support of many donors, including: the Bill \& Melinda Gates Foundation, the Ministry of Foreign Affairs of Denmark, Irish Aid, the Ministry of Finance of Japan in partnership with The World Bank, the Ministry of Foreign Affairs of the Netherlands, the Norwegian Agency for Development Cooperation (NORAD), the United Kingdom Department for International Development (DFID), and the United States Agency for International Development (USAID). The full list of IAVI donors is available at http://www.iavi.org). The contents of this manuscript are the responsibility of IAVI and do not necessarily reflect the views of USAID or the US Government.

Acknowledgements: We acknowledge the coordinated efforts and dedication of the Koome Youth Study research team and the Mukono district health office. We also thank Professor Wolfgang Weinmann, Dr. Joel Francis, Professor Alison Elliott and Dr. Judith Hahn for their scientific advice. Special thanks to Dr. Chrissy Roberts for supporting ODK and to Professor Wolfgang for overseeing PEth analysis at Bern University. We are indebted to all the fishing communities who agreed to participate in this study and grateful for the privilege of working with them.

Conflict of interest: The authors declare no conflict of interest

\section{References}

\section{References}

1. World Health Organisation. Global Status Report on Alcohol and Health. Geneva, Switzerland; World health organisation, Geneva, Switzerland 2018; pp. 1-43.

2. United Nations Office on Drug and Crime. World Drug Report. Availabe online: https://wdr.unodc.org/wdr2019/prelaunch/WDR19_Booklet_1_EXECUTIVE_SUMMARY.pdf (accessed on 27 June 2019).

3. Hall, W.D.; Patton, G.; Stockings, E.; Weier, M.; Lynskey, M.; Morley, K.I.; Degenhardt, L. Why young people's substance use matters for global health. Lancet Psychiatry 2016, 3, 265-279, doi:10.1016/s22150366(16)00013-4.

4. Degenhardt, L.; Stockings, E.; Patton, G.; Hall, W.D.; Lynskey, M. The increasing global health priority of substance use in young people. Lancet Psychiatry 2016, 3, 251-264.

5. McCall, C. Alcohol industry looks to boost drinks sales in Africa. Lancet 2017, 389, 589-590, doi:10.1016/S0140-6736(17)30330-6.

6. Degenhardt, L.; Peacock, A.; Colledge, S.; Leung, J.; Grebely, J.; Vickerman, P.; Stone, J.; Cunningham, E.B.; Trickey, A.; Dumchev, K.; et al. Global prevalence of injecting drug use and sociodemographic characteristics and prevalence of $\mathrm{HIV}, \mathrm{HBV}$, and $\mathrm{HCV}$ in people who inject drugs: a multistage systematic review. Lancet Glob. Health 2017, 5, e1192-e1207, doi:10.1016/s2214-109x(17)30375-3.

7. UNAIDS. Fact sheet-Latest statistics on the status of the AIDS epidemic. Availabe online: http://www.unaids.org/en/resources/fact-sheet (accessed on 9 April 2018).

8. Kuteesa, M.O.; Seeley, J.; Weiss, H.A.; Cook, S.; Kamali, A.; Webb, E.L. Alcohol misuse and illicit drug use among occupational groups at high risk of HIV in sub-Saharan Africa: A systematic review. AIDS Behav. 2019, doi:10.1007/s10461-019-02483-y. 
9. Hasin, D.S.; O'Brien, C.P.; Auriacombe, M.; Borges, G.; Bucholz, K.; Budney, A.; Compton, W.M.; Crowley, T.; Ling, W.; Petry, N.M.; et al. DSM-5 criteria for substance use disorders: recommendations and rationale. Am. J. Psychiatry 2013, 170, 834-851, doi:10.1176/appi.ajp.2013.12060782.

10. Seeley, J.; Nakiyingi-Miiro, J.; Kamali, A.; Mpendo, J.; Asiki, G.; Abaasa, A.; De Bont, J.; Nielsen, L.; Kaleebu, $P$. High HIV incidence and socio-behavioral risk patterns in fishing communities on the shores of Lake Victoria, Uganda. Sex. Transm. Dis. 2012, 39, 433-439.

11. Ssekandi, I.; Ssetaala, A.; Mpendo, J.; Nanvubya, A.; Nielsen, L.; Kiwanuka, N. Alcohol and illicit drug use among potential HIV vaccine efficacy trial volunteers along Lake Victoria, Uganda. Retrovirology 2012, 9, P219.

12. Kiene, S.M.; Lule, H.; Sileo, K.M.; Silmi, K.P.; Wanyenze, R.K. Depression, alcohol use, and intimate partner violence among outpatients in rural Uganda: vulnerabilities for HIV, STIs and high risk sexual behavior. BMC Infect. Dis. 2017, 17, 88.

13. Tumwesigye, N.M.; Atuyambe, L.; Wanyenze, R.K.; Kibira, S.P.; Li, Q.; Wabwire-Mangen, F.; Wagner, G. Alcohol consumption and risky sexual behaviour in the fishing communities: evidence from two fish landing sites on Lake Victoria in Uganda. BMC Public Health 2012, 12, 1.

14. Kiwanuka, N.; Ssetaala, A.; Nalutaaya, A.; Mpendo, J.; Wambuzi, M.; Nanvubya, A.; Sigirenda, S.; Kitandwe, P.K.; Nielsen, L.E.; Balyegisawa, A. High incidence of HIV-1 infection in a general population of fishing communities around Lake Victoria, Uganda. PLoS ONE 2014, 9, e94932.

15. Smolak, A. A meta-analysis and systematic review of HIV risk behavior among fishermen. AIDS Care 2014, 26, 282-291.

16. Kwena, Z.A.; Camlin, C.S.; Shisanya, C.A.; Mwanzo, I.; Bukusi, E.A. Short-term mobility and the risk of $\mathrm{HIV}$ infection among married couples in the fishing communities along Lake Victoria, Kenya. PLoS ONE 2013, 8, e54523.

17. Sileo, K.M.; Kintu, M.; Chanes-Mora, P.; Kiene, S.M. “Such Behaviors Are Not in My Home Village, I Got Them Here": A Qualitative Study of the Influence of Contextual Factors on Alcohol and HIV Risk Behaviors in a Fishing Community on Lake Victoria, Uganda. AIDS Behav. 2016, 20, 537-547.

18. Kiwanuka, N.; Ssetaala, A.; Ssekandi, I.; Nalutaaya, A.; Kitandwe, P.K.; Ssempiira, J.; Bagaya, B.S.; Balyegisawa, A.; Kaleebu, P.; Hahn, J.; et al. Population attributable fraction of incident HIV infections associated with alcohol consumption in fishing communities around Lake Victoria, Uganda. PLoS ONE 2017, 12, e0171200, doi:10.1371/journal.pone.0171200.

19. Sanya, R.E.; Nkurunungi, G.; Hoek Spaans, R.; Nampijja, M.; O’Hara, G.; Kizindo, R.; Oduru, G.; Kabuubi Nakawungu, P.; Niwagaba, E.; Abayo, E. The Impact of Intensive Versus Standard Anthelminthic Treatment on Allergy-related Outcomes, Helminth Infection Intensity, and Helminth-related Morbidity in Lake Victoria Fishing Communities, Uganda: Results From the LaVIISWA Cluster-randomized Trial. Clin. Infect. Dis. 2019, 68, 1665-1674.

20. Sanya, R.E.; Muhangi, L.; Nampijja, M.; Nannozi, V.; Nakawungu, P.K.; Abayo, E.; Webb, E.L.; Elliott, A.M. Schistosoma mansoni and HIV infection in a Ugandan population with high HIV and helminth prevalence. Trop. Med. Int. Health 2015, 20, 1201-1208.

21. Francis, J.M.; Grosskurth, H.; Changalucha, J.; Kapiga, S.H.; Weiss, H.A. Systematic review and metaanalysis: prevalence of alcohol use among young people in eastern Africa. Trop. Med. Int. Health 2014, 19, 476-488.

22. Babor, T.F.; Higgins-Biddle, J.C.; Saunders, J.B.; Monteiro, M.G. The Alcohol Use Disorders Identification Test: Guidelines for Use in Primary Care; World Health Organization: Geneva, Switzerland, 2001.

23. Sobell, L.; Sobell, M. Timeline Followback: A Technique for Assessing Self Reported Ethanol Consumption; Humana Press: Totowa, NJ, USA, 1992; Volume 17.

24. Helander, A.; Zheng, Y. Molecular species of the alcohol biomarker phosphatidylethanol in human blood measured by LC-MS. Clin. Chem. 2009, 55, 1395-1405, doi:10.1373/clinchem.2008.120923.

25. Hahn, J.A.; Dobkin, L.M.; Mayanja, B.; Emenyonu, N.I.; Kigozi, I.M.; Shiboski, S.; Bangsberg, D.R.; Gnann, H.; Weinmann, W.; Wurst, F.M. Phosphatidylethanol (PEth) as a biomarker of alcohol consumption in HIVpositive patients in sub-Saharan Africa. Alcohol. Clin. Exp. Res. 2012, 36, 854-862.

26. Degenhardt, L.; Hall, W.; Warner-Smith, M.; Lynskey, M. Illicit drug use. In Comparative Quantification of Health Risks: Global and Regional Burden of Disease Attributable to Selected Major Risk Factors; World Health organisation: Geneva 2004; Volume 1, pp. 1109-1176. 
27. World Health Organisation ASSIST Working Group. The Alcohol, Smoking and Substance Involvement Screening Test (ASSIST): development, reliability and feasibility. Addict. (Abingdon Engl.) 2002, 97, 11831194.

28. Wurst, F.M.; Thon, N.; Aradottir, S.; Hartmann, S.; Wiesbeck, G.A.; Lesch, O.; Skala, K.; Wolfersdorf, M.; Weinmann, W.; Alling, C. Phosphatidylethanol: normalization during detoxification, gender aspects and correlation with other biomarkers and self-reports. Addict. Biol. 2010,15, 88-95.

29. Viel, G.; Boscolo-Berto, R.; Cecchetto, G.; Fais, P.; Nalesso, A.; Ferrara, S. Phosphatidylethanol in blood as a marker of chronic alcohol use: a systematic review and meta-analysis. Int. J. Mol. Sci. 2012, 13, 1478814812.

30. Kroenke, K.; Spitzer, R.L.; Williams, J.B. The PHQ-9: validity of a brief depression severity measure. J. Gen. Intern. Med. 2001, 16, 606-613, doi:10.1046/j.1525-1497.2001.016009606.x.

31. Hill-Kapturczak, N.; Dougherty, D.M.; Roache, J.D.; Karns-Wright, T.E.; Lopez-Cruzan, M.; Javors, M.A. Chapter 58-Phosphatidylethanol Homologs in Blood as Biomarkers for the Time Frame and Amount of Recent Alcohol Consumption. In Neuroscience of Alcohol; Preedy, V.R., Ed. Academic Press: Cambridge, MA, USA, 2019; doi:10.1016/B978-0-12-813125-1.00058-1pp. 567-576.

32. Helander, A.; Hansson, T. National harmonization of the alcohol biomarker PEth. Lakartidningen 2013, 110, 1747.

33. Ministry of health Uganda. National HIV Testing Services Policy and Implementation Guidelines; Uganda Ministry of Health: Kampala 2016.

34. Victora, C.G.; Huttly, S.R.; Fuchs, S.C.; Olinto, M.T. The role of conceptual frameworks in epidemiological analysis: a hierarchical approach. Int. J. Epidemiol. 1997, 26, 224-227, doi:10.1093/ije/26.1.224.

35. Francis, J.M.; Weiss, H.A.; Mshana, G.; Baisley, K.; Grosskurth, H.; Kapiga, S.H. The epidemiology of alcohol use and alcohol use disorders among young people in northern Tanzania. PLoS ONE 2015, 10, e0140041.

36. Verenkar, Y.J.; Vaz, F.S. Prevalence and pattern of alcohol consumption using alcohol use disorder identification test among students at a medicalcollege in Goa, India. Int. J. Community Med. Public Health 2018, 5, 2935-2938.

37. Weiss, H.A.; Vandepitte, J.; Bukenya, J.N.; Mayanja, Y.; Nakubulwa, S.; Kamali, A.; Seeley, J.; Grosskurth, $\mathrm{H}$. High levels of persistent problem drinking in women at high risk for HIV in Kampala, Uganda: A prospective cohort study. Int. J. Environ. Res. Public Health 2016, 13, 153.

38. Korhonen, C.; Kimani, M.; Wahome, E.; Otieno, F.; Okall, D.; Bailey, R.C.; Harper, G.W.; Lorway, R.R.; Doshi, M.; Mathenge, J. Depressive symptoms and problematic alcohol and other substance use in 1476 gay, bisexual, and other MSM at three research sites in Kenya. AIDS (London, Engl.) 2018, 32, 1507.

39. Peltzer, K.; Pengpid, S. Correlates of illicit drug use among university students in Africa and the Caribbean. J. Psychol. Afr. 2016, 26, 390-393.

40. Haug, S.; Nunez, C.L.; Becker, J.; Gmel, G.; Schaub, M.P. Predictors of onset of cannabis and other drug use in male young adults: results from a longitudinal study. BMC Public Health 2014, 14, 1202, doi:10.1186/14712458-14-1202.

41. Howell, A.N.; Leyro, T.M.; Hogan, J.; Buckner, J.D.; Zvolensky, M.J. Anxiety sensitivity, distress tolerance, and discomfort intolerance in relation to coping and conformity motives for alcohol use and alcohol use problems among young adult drinkers. Addict. Behav. 2010, 35, 1144-1147.

42. MacPherson, L.; Magidson, J.F.; Reynolds, E.K.; Kahler, C.W.; Lejuez, C.W. Changes in Sensation Seeking and Risk-Taking Propensity Predict Increases in Alcohol Use Among Early Adolescents. Alcohol. Clin. Exp. Res. 2010, 34, 1400-1408, doi:10.1111/j.1530-0277.2010.01223.x.

43. Pandrea, I.; Happel, K.I.; Amedee, A.M.; Bagby, G.J.; Nelson, S. Alcohol's role in HIV transmission and disease progression. Alcohol. Res. Health, 2010,33, 203-218.

44. Reid, S.R. Injection drug use, unsafe medical injections, and HIV in Africa: a systematic review. Harm Reduct. J. 2009, 6, 24.

45. Hendershot, C.S.; Stoner, S.A.; Pantalone, D.W.; Simoni, J.M. Alcohol use and antiretroviral adherence: review and meta-analysis. J. Acquir. Immune Defic. Syndr. (1999) 2009, 52, 180.

46. Looker, K.J.; Elmes, J.A.R.; Gottlieb, S.L.; Schiffer, J.T.; Vickerman, P.; Turner, K.M.E.; Boily, M.C. Effect of HSV-2 infection on subsequent HIV acquisition: an updated systematic review and meta-analysis. Lancet Infect. Dis. 2017, 17, 1303-1316, doi:10.1016/s1473-3099(17)30405-x. 
47. Rothman, K.J.; Greenland, S.; Lash, T.L. Modern Epidemiology; Wolters Kluwer Health/Lippincott Williams \& Wilkins: Philadelphia, USA: 2008; Volume 3.

48. Morton, L.M.; Cahill, J.; Hartge, P. Reporting Participation in Epidemiologic Studies: A Survey of Practice. Am. J. Epidemiol. 2005, 163, 197-203, doi:10.1093/aje/kwj036.

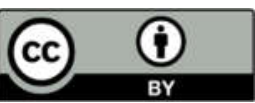

(C) 2020 by the authors. Licensee MDPI, Basel, Switzerland. This article is an open access article distributed under the terms and conditions of the Creative Commons Attribution (CC BY) license (http://creativecommons.org/licenses/by/4.0/). 